\title{
Determination of Dyclonine Hydrochloride by a HPLC Method and Camphor and Menthol by a GC Method in Compound Lotion
}

\author{
Suying Ma, Haixia Lv, and Xiaojun Shang \\ School of Pharmacy, Xinxiang Medical University, Xinxiang 453003, China \\ Correspondence should be addressed to Suying Ma; suyingma@163.com
}

Received 22 June 2012; Accepted 6 August 2012

Academic Editor: Eulogio J. Llorent-Martinez

Copyright (C) 2013 Suying Ma et al. This is an open access article distributed under the Creative Commons Attribution License, which permits unrestricted use, distribution, and reproduction in any medium, provided the original work is properly cited.

\begin{abstract}
A high performance liquid chromatographic (HPLC) method with UV detector for the determination of dyclonine hydrochloride and a gas chromatography (GC) method with flame ionization detector (FID) for the determination of camphor and menthol in lotion were developed. The developed HPLC method involved using a SinoChoom ODS-BP $\mathrm{C}_{18}$ reversed-phase column $(5 \mu \mathrm{m}$, $4.6 \mathrm{~mm} \times 200 \mathrm{~mm}$ ) and mobile phase consisting of acetonitrile : water : triethylamine in a ratio of $45: 55: 1.0$; $\mathrm{pH}$ was adjusted to 3.5 with glacial acetic acid. The developed GC method for determination of camphor and menthol involved using an Agilent 19091J-413 capillary chromatographic column $(30 \mathrm{~m} \times 320 \mu \mathrm{m} \times 0.25 \mu \mathrm{m})$. The two methods were validated according to official compendia guidelines. The calibration of dyclonine hydrochloride for HPLC method was linear over the range of $20-200 \mu \mathrm{g} / \mathrm{mL}$. The retention time was found at $6.0 \mathrm{~min}$ for dyclonine hydrochloride. The calibration of camphor and menthol of GC method was linear over the range of $10-2000 \mu \mathrm{g} / \mathrm{mL}$. The retention time was found at $2.9 \mathrm{~min}$ for camphor and $3.05 \mathrm{~min}$ for menthol. The proposed HPLC and GC methods were proved to be suitable for the determination of dyclonine hydrochloride, camphor, and menthol in lotion.
\end{abstract}

\section{Introduction}

Dyclonine hydrochloride (Figure 1(a)), chemically 1-(4-butoxyphenyl)-3-1-piperidinyl)-1-ropanon hydrochloride is belonging to a bactericidal and fungicidal local anesthetic. It has already become an important drug in the treatment of alleviation pain and antipruritic $[1,2]$. Menthol (Figure 1(b)), chemically (1R,2S,5R)-2-isopropyl-5-methylcyclohexanol, is an organic compound made synthetically or isolated from Mentha haplocalyx Briq. which is an important medicine documented in Chinese Pharmacopoeia and has been widely used as an antipyretic and analgesic agent for treatment of headaches and itching [3, 4]. Camphor (Figure 1(c)), chemically 1,7,7-trimethyl bicyclo [2.2.1] heptan-2-one, is isolated from Cinnamomum camphora (L.) Sieb. which is mainly distributed in the south of the Yangtze River, and now it can be synthesized by chemist. It was used for agi-tation of the central nervous system and enhancement of the respiratory and circulatory system. It could also be used for alleviating pain and antipruritic [5-9].
The compound lotion is prepared with dyclonine hydrochloride, menthol, and camphor. The preparation is accepted by patients for many advantages such as less grease, skin cool, and so on. To the best of our knowledge, the current assay method was mainly focused on dyclonine hydrochloride; few methods have been developed in the literature for the estimation of dyclonine hydrochloride, menthol and camphor in preparations $[10,11]$. The aim of this study is an attempt to develop and validate an HPLC method for dyclonine hydrochloride and a GC method for menthol and camphor with the advantages of shorter retention time and run time.

\section{Experimental}

Pure dyclonine hydrochloride, camphor, and menthol used as working standards were received as gifts from Zhengzhou Yonghe Pharmaceuticals Co., Ltd., Zhengzhou, China. Acetonitrile of HPLC grade and other chemicals of AR grade were purchased from Mingshen Chemicals Co., Ltd., Zhengzhou, 
<smiles>CCCCOc1ccc(C(=O)CCN2CCCCC2)cc1</smiles>

(a)



(b)



(c)

FIGURE 1: Typical chemical structures of (a) dyclonine hydrochloride, (b) menthol, and (c) camphor.

China. Deionized water was obtained by using a Milli-Q water purification system (Millipore, Bedford, MA, USA).

\subsection{Preparation of Standard Stock Solution and Working} Solution. The standard solution of dyclonine hydrochloride was prepared by dissolving accurately $10 \mathrm{mg}$ pure drug in a $10 \mathrm{~mL}$ volumetric flask using mobile phase. The solution was sonicated for $5 \mathrm{~min}$ and then made up to the mark with mobile phase. Intermediate and working solutions were prepared by diluting stock solutions with the mobile phase.

The combined standard solution of menthol and camphor was prepared by dissolving accurately $10 \mathrm{mg}$ menthol and $10 \mathrm{mg}$ camphor in a $10 \mathrm{~mL}$ volumetric flask using chloroform. The solution was sonicated for $5 \mathrm{~min}$ and then made up to the mark with chloroform. Intermediate and working solutions were prepared by diluting stock solutions with chloroform.

2.2. HPLC Determination Conditions. Chromatographic analyses were performed using a Shimadzu system that was comprised of an LC-20AT pump, SPD 20A UV-Visible absorbance detector connected to Shimadzu Spin Chrome software. ODS-BP $C_{18}$ column $(5 \mu \mathrm{m}, 4.6 \mathrm{~mm} \times 200 \mathrm{~mm})$ was used and the sample injection was performed via a Rheodyne syringe.

The mobile phase was a mixture of acetonitrile : water : triethylamine in a ratio of $45: 55: 1.0$. The $\mathrm{pH}$ of mobile phase was adjusted to 3.5 with glacial acetic acid. The flow rate was $1.0 \mathrm{~mL} / \mathrm{min}$. The mobile phase was degassed by an ultrasonic bath and filtered through a $0.45 \mu \mathrm{m}$ membrane filter under vacuum. Wavelength was set at $280 \mathrm{~nm}$. Flow rate was kept at $1.0 \mathrm{~mL} / \mathrm{min}$. The column was maintained at $35^{\circ} \mathrm{C}$ and injection volume was $20 \mu \mathrm{L}$.

2.3. GC Determination Conditions. The GC method developed for determination of camphor and menthol was performed with Agilent 7890A and separated on Agilent $19091 \mathrm{~J}-413$ fused capillary chromatographic column $(30 \mathrm{~m} \times$ $320 \mu \mathrm{m} \times 0.25 \mu \mathrm{m}$ ) which was coupled to an FID detector. The temperature of detector and inlet was maintained at $250^{\circ} \mathrm{C}$. The oven temperature was programmed at $250^{\circ} \mathrm{C}$ for $1 \mathrm{~min}$, then $5^{\circ} \mathrm{C} / \mathrm{min}$ to $170^{\circ} \mathrm{C}$, and then hold at $170^{\circ} \mathrm{C}$ for $5 \mathrm{~min}$. The split ratio was $10: 1$. The carrier gas was nitrogen
(99.99\% purity) with a flow rate of $1.5 \mathrm{~mL} / \mathrm{min}$, and the analyzed sample volume was $1 \mu \mathrm{L}$.

2.4. Method Validation. The method was validated as per ICH guidelines for specificity, linearity, accuracy, precision, and reproducibility.

2.4.1. Specificity. Dyclonine hydrochloride working solution $(20.0 \mu \mathrm{g} / \mathrm{mL})$ and excipients sample working solution (including camphor $40.0 \mu \mathrm{g} / \mathrm{mL}$ and menthol $40.0 \mu \mathrm{g} / \mathrm{mL}$ ) were scanned from $200 \mathrm{~nm}$ to $400 \mathrm{~nm}$, and then the chromatograms were recorded with HPLC system.

Combined working solution (including camphor $80.0 \mu \mathrm{g} /$ $\mathrm{mL}$ and menthol $80.0 \mu \mathrm{g} / \mathrm{mL}$ ) and excipients sample working solution (including dyclonine hydrochloride $40.0 \mu \mathrm{g} / \mathrm{mL}$ ) were recorded with GC system.

2.4.2. Linearity. The linearity of the HPLC method for dyclonine hydrochloride was determined at seven concentration levels. The linearity of the GC method for camphor and menthol was determined at ten concentration levels. The responses were measured as peak areas.

2.4.3. Accuracy. A standard addition method was employed for accuracy experiment. Adequate amounts of dyclonine hydrochloride, camphor, and menthol corresponding to $80 \%$, $100 \%$, and $120 \%$ of the claimed concentration levels were added to excipients. At each level, three determinations were performed and the results were recorded. Accuracy was expressed as percent analyte recovered by the two proposed methods.

2.4.4. Precision. The precision of the two methods was checked by repeatability of injection, repeatability (intraday), intermediate precision (interday), and reproducibility. The results were expressed as the percentage relative standard deviation (\% RSD) for ten determinations of peak areas of dyclonine hydrochloride $(20.0 \mu \mathrm{g} / \mathrm{mL})$ and camphor and menthol $(80.0 \mu \mathrm{g} / \mathrm{mL})$ performed. The same solutions were injected in triplicate for both intraday and interday variation.

2.5. Method Applications. $1 \mathrm{~mL}$ compound lotion was accurately transferred into another $50 \mathrm{~mL}$ volumetric flask and 


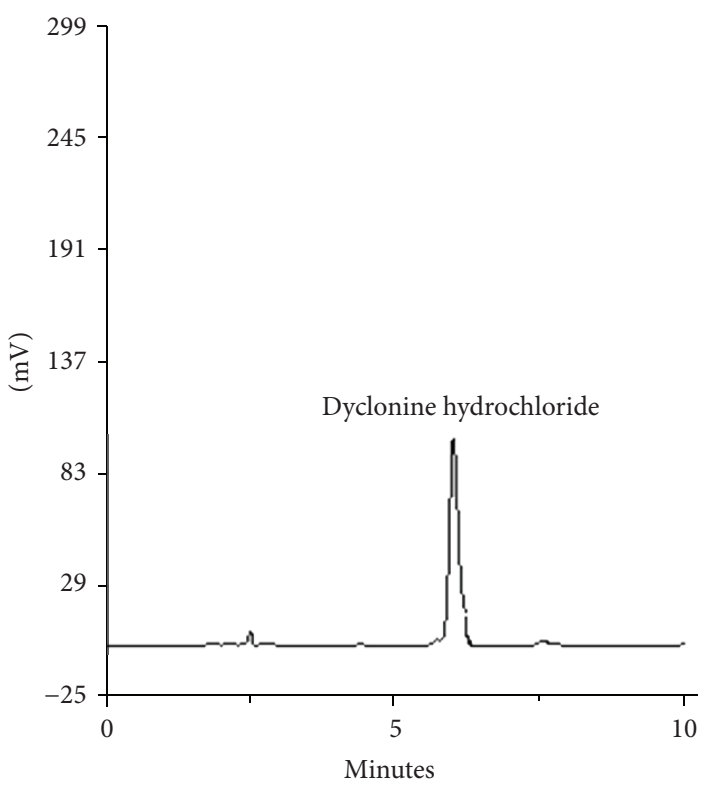

(a)

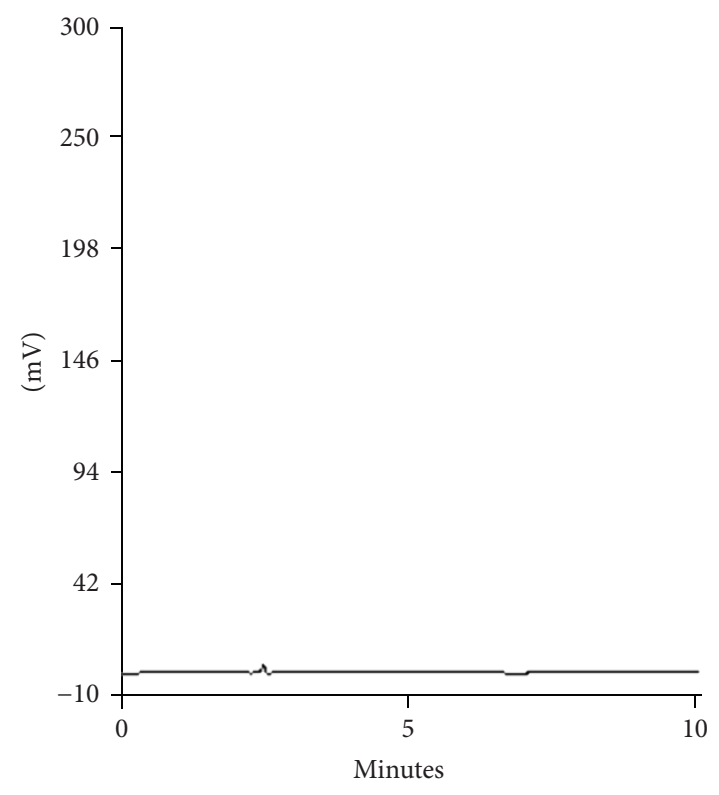

(b)

FIgURE 2: Typical HPLC chromatograms of dyclonine hydrochloride $(20.0 \mu \mathrm{g} / \mathrm{mL})$, excipients sample working solution (including camphor $40.0 \mu \mathrm{g} / \mathrm{mL}$ and menthol $40.0 \mu \mathrm{g} / \mathrm{mL})$.

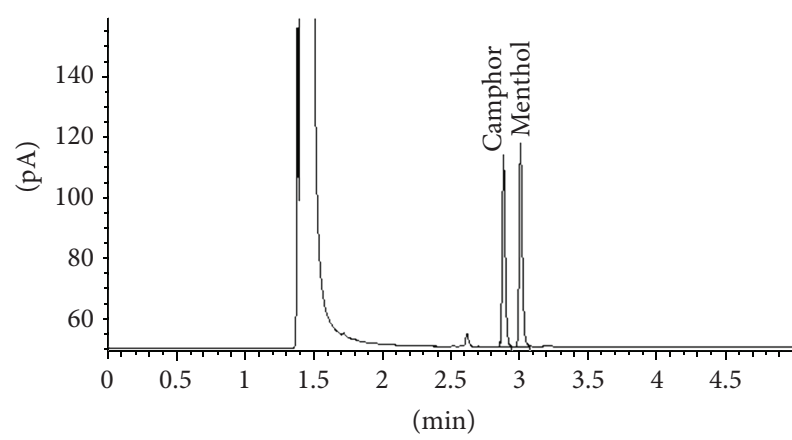

(a)

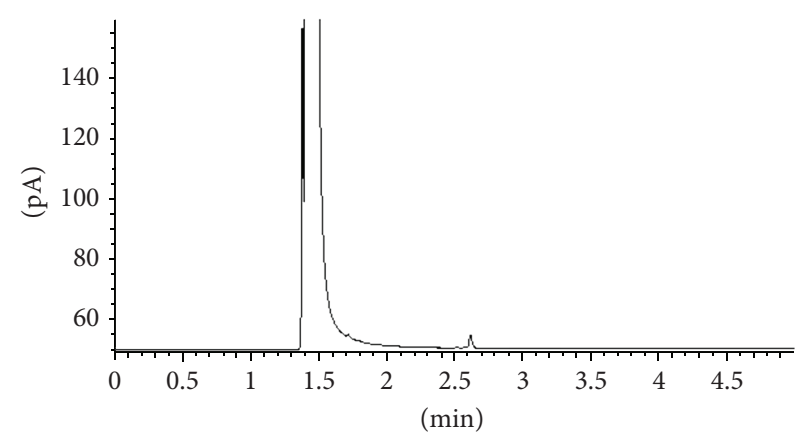

(b)

FIGURE 3: Typical GC chromatograms of camphor $(80.0 \mu \mathrm{g} / \mathrm{mL})$ and menthol $(80.0 \mu \mathrm{g} / \mathrm{mL})$, excipients sample working solution (including dyclonine hydrochloride $40.0 \mu \mathrm{g} / \mathrm{mL}$ ).

made up to volume with mobile phase to yield concentrations of $100 \mu \mathrm{g} / \mathrm{mL}$ for dyclonine hydrochloride. A $20 \mu \mathrm{L}$ volume of the sample solution was injected into the chromatographic system three times under optimized HPLC conditions. The concentrations in the samples were determined by interpolation from calibration plots of dyclonine hydrochloride previously obtained.

$1 \mathrm{~mL}$ compound lotion was accurately transferred into another $10 \mathrm{~mL}$ glass tube, and $5 \mathrm{~mL}$ chloroform was used to dilute the solution. The mixture was shaken for $2 \mathrm{~min}$ and centrifuged at $3000 \mathrm{rpm}$ for $5 \mathrm{~min}$. The above procedure was repeated twice. The supernatant was combined and evaporated to dryness at $50^{\circ} \mathrm{C}$ in bath water. The residue was dissolved with $10 \mathrm{~mL}$ chloroform. A $1 \mu \mathrm{L}$ volume of the sample solution was injected into the chromatographic system three times under optimized GC conditions. The concentrations in the samples were determined by interpolation from calibration plots of camphor and menthol previously obtained.

\section{Results and Discussion}

3.1. Specificity. The chromatographic conditions were optimized. According to the ultraviolet spectroscopy, dyclonine hydrochloride has maximum absorbance at $280 \mathrm{~nm}$. Thus, $280 \mathrm{~nm}$ was selected as detected wavelength. Under the optimum conditions, typical chromatograms of dyclonine hydrochloride, camphor, menthol, and blank excipients are shown in Figures 2 and 3. The analyte peaks were well resolved and free from tailing ( $<1.5$ for all analytes). The excipients in the compound lotion did not disturb the detection in the two methods. The retention time of dyclonine hydrochloride was found to be $6.0 \mathrm{~min}$ in HPLC chromatogram. The retention times of camphor and menthol 
were found to be $2.9 \mathrm{~min}$ and $3.05 \mathrm{~min}$ in GC chromatogram.

3.2. Linearity. Under the optimal conditions, the HPLC method was linear over the range $20-200 \mu \mathrm{g} / \mathrm{mL}$ for dyclonine hydrochloride. The regression equation $Y=67.94 X-$ 157.56 was established based on the standard samples injected and their peak area with correlation coefficient of 0.9999 .

The GC method was linear over the range $20-2000 \mu \mathrm{g} / \mathrm{mL}$ for camphor and menthol. The regression equation $Y=$ $1.7359 X-39.406$ for menthol and $Y=1.5687 X-35.544$ for camphor was established based on the standard samples injected and their peak area with correlation coefficient of 0.9991 and 0.9993 .

The results indicate an excellent correlation between response factor and concentration of the three drugs.

3.3. Accuracy. The mean recovery data from the study for dyclonine hydrochloride was $99.92 \%$ in HPLC method, while they were $99.47 \%$ and $100.05 \%$ for camphor and menthol in GC method.

3.4. Precision. The precision study of intra- and interday variability was performed to determine the precision of the two developed methods. The RSD of intraday variation was $0.66 \%$ and the RSD of interday variation was $0.73 \%$ for dyclonine hydrochloride, respectively. The injection repeatability value of dyclonine hydrochloride was $0.89 \%$ in HPLC method.

The RSD of intraday variation was $0.49 \%$ and the RSD of interday variation was $0.51 \%$ for camphor; they were $0.72 \%$ and $0.83 \%$ for menthol, respectively. The injection repeatability values were $0.63 \%$ and $0.66 \%$ for camphor and menthol in GC method, respectively.

The results indicate that the two methods are precise and reproducible.

3.5. Method Applications. The validated two methods were applied to the determination of dyclonine hydrochloride, camphor, and menthol in compound lotion. The results of the assay yielded $99.43 \pm 0.28 \%, 100.08 \pm 0.36 \%$, and $99.57 \pm$ $0.45 \%$ for dyclonine hydrochloride, camphor, and menthol, respectively. It showed that the two methods were selective for determination of dyclonine hydrochloride, camphor, and menthol without interference from the excipients used in the compound lotion.

\section{Conclusion}

It is concluded from the above study that the two developed methods were simple, high sensitive and provide good reproducibility and accurateness for the determination of dyclonine hydrochloride, camphor, and menthol in compound lotion.

\section{Acknowledgments}

The authors are very much thankful to Xinxiang Medical University for financial support and Zhengzhou Yonghe
Pharmaceuticals Co., Ltd., for providing the free gift samples of working standards.

\section{References}

[1] http://en.wikipedia.org/wiki/Dyclonine.

[2] T. Li, C. Li, K. L. Zhang, F. L. Jie, Y. W. Luo, and L. J. Chen, "Preparation and quality control of compand dyclonine menthol antipruritic emollients lotion," China Pharmacy, vol. 22, no. 21, pp. 1965-1967, 2011.

[3] H. L. Fang, W. L. Li, B. R. Ren, Y. Ch. Liang, and Y. Liu, "Research advance on chemical constituents and pharmacological activities of mentha L," China Pharmaceuticals, vol. 19, no. 10, pp. 13-17, 2010.

[4] http://en.wikipedia.org/wiki/Menthol.

[5] J. Pauwels, W. Daury, L. Van den Bossche et al., "Optimization and validation of liquid chromatography and headspace-gas chromatography based methods for the quantitative determination of capsaicinoids, salicylic acid, glycol monosalicylate, methyl salicylate, ethyl salicylate, camphor and l-enthol in a topical formulation," Journal of Pharmaceutical and Biomedical Analysis, vol. 60, pp. 51-58, 2012.

[6] http://en.wikipedia.org/wiki/Camphor.

[7] X. W. He, F. C. Zhang, and Y. Jiang, "An improved ionic liquidbased headspace single-drop microextraction-liquid chromatography method for the analysis of camphor and transanethole in compound liquorice tablets," Journal of Chromatographic Science, no. 50, pp. 457-463, 2012.

[8] K. Schulz, M. Bertau, K. Schlenz, S. Malt, J. Dreßler, and D. W. Lachenmeier, "Headspace solid-phase microextraction-gas chromatography-mass spectrometry determination of the characteristic flavourings menthone, isomenthone, neomenthol and menthol in serum samples with and without enzymatic cleavage to validate post-offence alcohol drinking claims," Analytica Chimica Acta, vol. 646, no. 1-2, pp. 128-140, 2009.

[9] Y. Sitaramaraju, A. van Hul, K. Wolfs, A. Van Schepdael, J. Hoogmartens, and E. Adams, "Static headspace gas chromatography of (semi-)volatile drugs in pharmaceuticals for topical use," Journal of Pharmaceutical and Biomedical Analysis, vol. 47, no. 4-5, pp. 834-840, 2008.

[10] Z. H. Li and Y. L. Gao, "Determination of principal agents and the related substance of compound ftibamzone/ dyclonine liniment by HPLC," China Pharmacy, vol. 20, no. 34, pp. 2697-2699, 2009.

[11] C. Li, H. Yan, and Y. W. Luo, "Dylonine content in the compound dyclonine menthol antipruritic emollients water with HPLC," Journal of Hainan Medical, vol. 21, no. 14, pp. 125-127, 2010. 

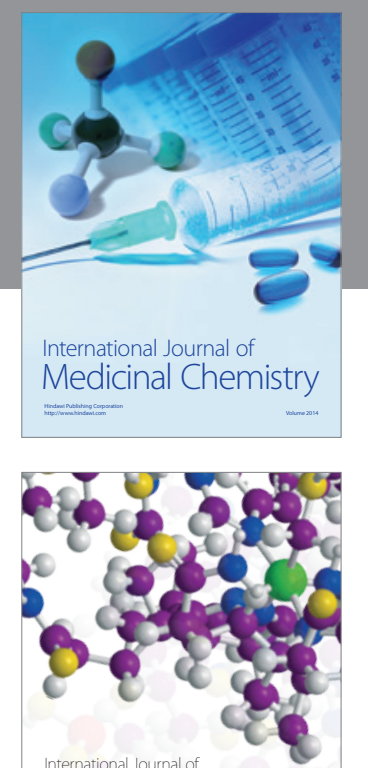

\section{Carbohydrate} Chemistry

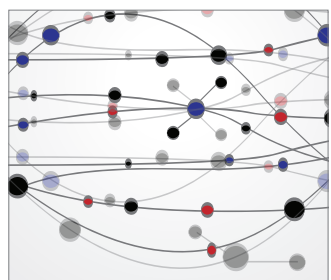

The Scientific World Journal
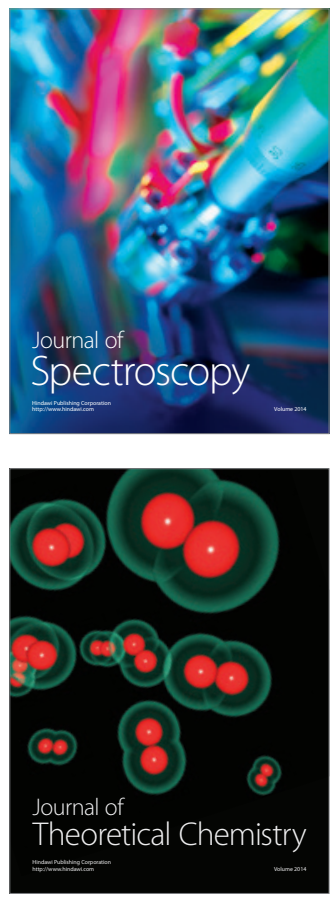
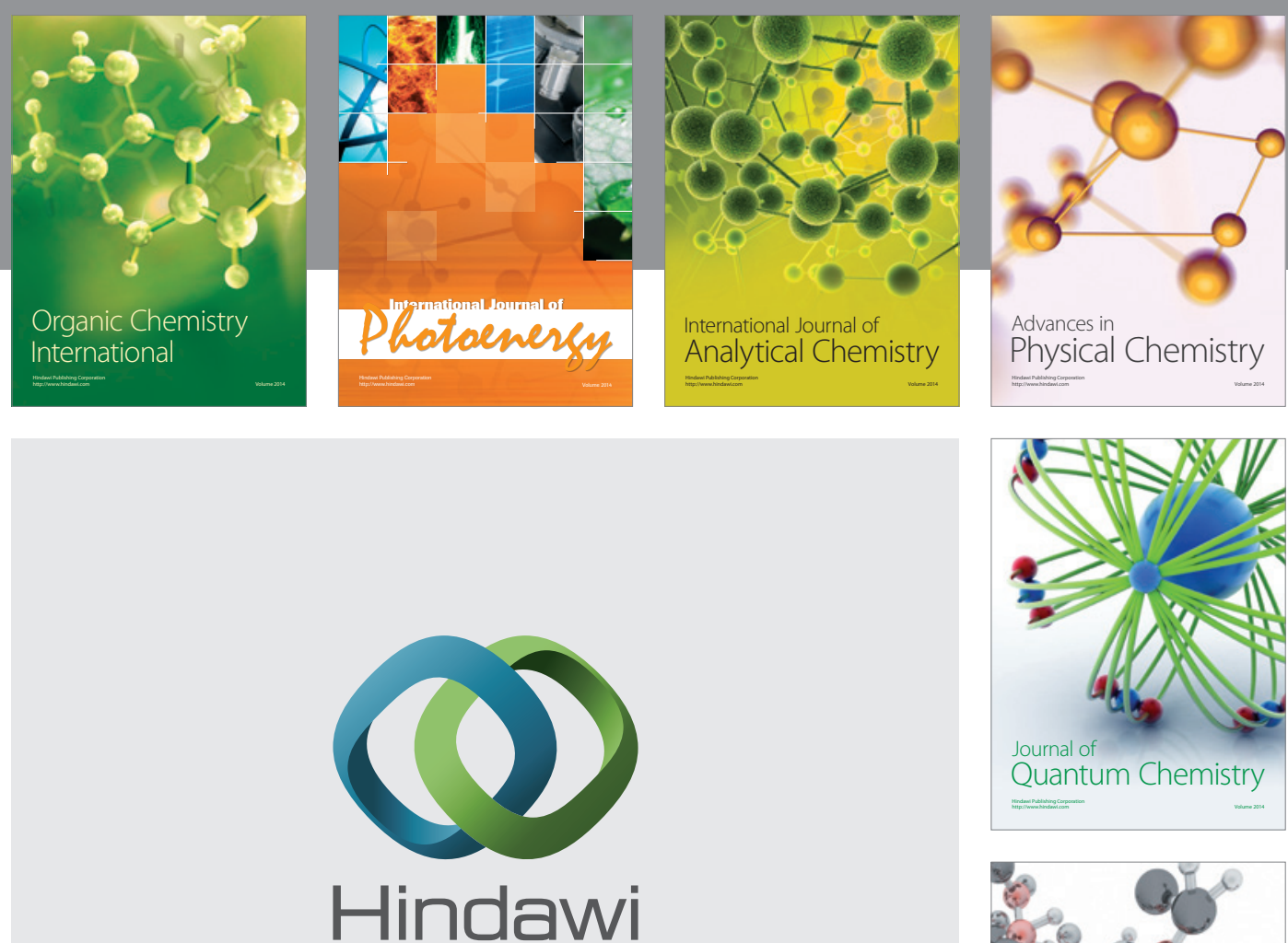

Submit your manuscripts at

http://www.hindawi.com

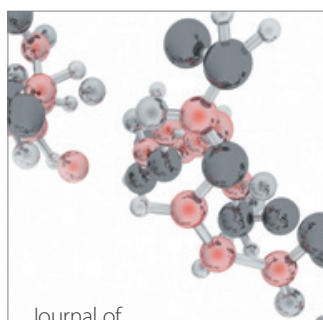

Analytical Methods

in Chemistry

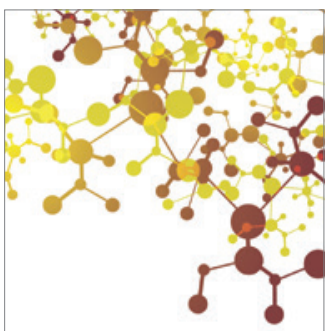

Journal of

Applied Chemistry



Inorganic Chemistry
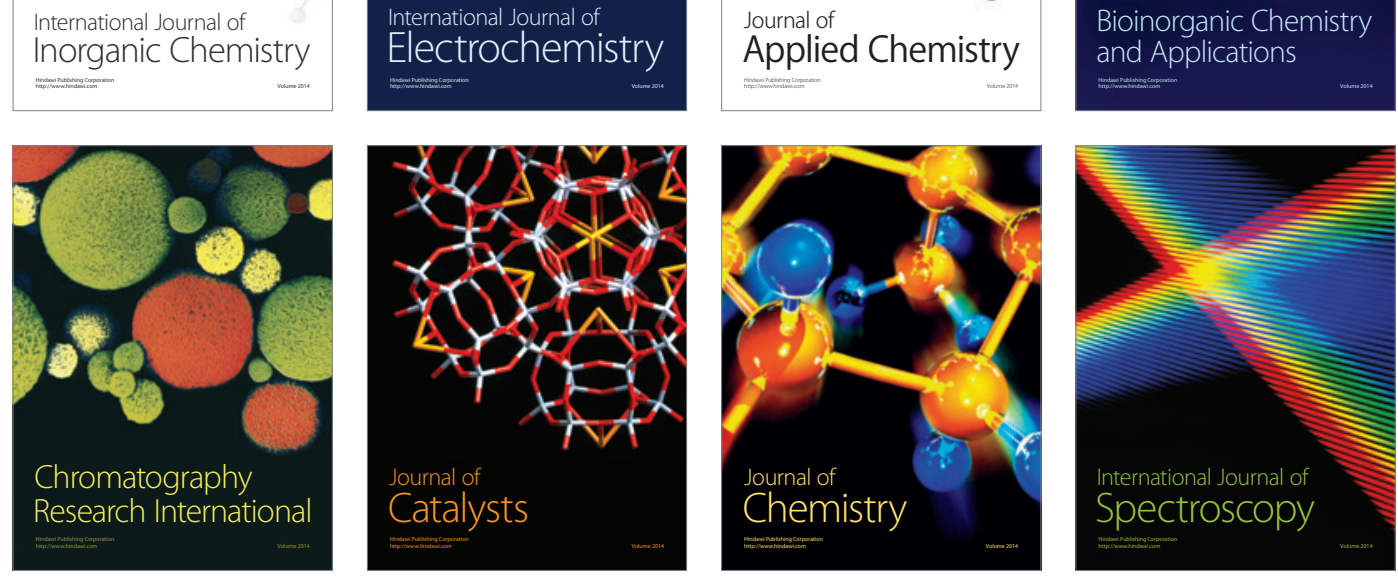